\title{
Interactive Three-Dimensional Model Reconstruction of Ultrasonography
}

\author{
Kritpanu Sopabutr ${ }^{\mathrm{a},},{ }^{,}$Paramate Horkaew ${ }^{\mathrm{a}}$, Duangrat Gansawat ${ }^{\mathrm{b}}$ \\ ${ }^{a}$ School of Computer Engineering, Suranaree University of Technology, Nakhonrachjasima 30000, Thailand \\ ${ }^{b}$ Image Technology Laboratory, National Electronics and Computer Technology Center,Pathumthani 12120, Thailand \\ *Corresponding Author:kritpanu.sopabutr@gmail.com
}

\begin{abstract}
This paper discusses interactive 3D reconstruction of ultrasonography model. The proposed scheme can be divided into three modules, consisting of Image Segmentation, 3D Model Reconstruction and Interactive Model Editing.More specifically, a set of 2D radial scans acquired from ultrasonography were segmented using Active Contour, resulting in an unorganized set of 3D points delineating an object of interest on multi-planar images. These points were subsequently fitted with a 3D surface model by an expansion of Spherical Harmonics. As accurately segmenting ultrasound images with low SNR can be challenging, this fitted model will be later interactively adjusted to be more properly coincided with underlying structure subject to expert's opinion. The contribution of this paper is therefore the algorithm and the organizing of related data structure which lend to responsive rendition of the parametric surface being edited.
\end{abstract}

Keywords: Image segmentation, active contour, surface reconstruction, spherical harmonics, interactive surface editing

\section{Introduction}

Image processing has become a primary step toward various computational vision schemes ${ }^{(1)}$. Most prominent ones include image enhancement, noise filtering and object segmentation for subsequent data analyses, e.g., size and shape estimation as well as motion detection ${ }^{(2)}$. Medical Imaging has also enjoyed these advancements. Not until recently, various imaging modalities such as the Computed Tomography (CT) and Ultrasonography had been primarily used as supplement data in visual diagnosis. Representation was merely in 2D grayscale image array, despite its full 3D modal capability. Modern visualization techniques such as surface reconstruction has made it possible for cutting edge scanners to produce 3D rendering of anatomical structures, which however remains inaccessible to less equipped clinic or local healthcare providers. This factor has thus impeded otherwise efficient communication between physicians and their patients regarding the understanding of the symptom, diagnosis and treatments. In particular for ultrasonography, for instance, real-time 3D visualization offers efficient and early prognostic determinant of the fetal anomalies in vivo. Existing built-in software nonetheless, mostly render the 3D structure by using voxel-based techniques, e.g., ray casting, etc. Despite its visual appearance, these non-photo realistic rendering limits their applications within the scope of visual assessment. Quantitative evaluation, on the other hand, would require yet an additional set of tools for drawing the geometrical overlaid, whose dimensions (length, area and volumes) may be calculated, to determine the degree of those anomalies. Explicit geometrical model reconstruction from various imaging modalities has attracted considerable interests in the literatures. Geometrical augmentation was proposed in a Virtual Reality scheme for surgical planning ${ }^{(3)}$ and other medical applications ${ }^{(4)}$, such as interactive colonoscopy system ${ }^{(5)}$. More recently, physical modeling was also incorporated into 3D synthetic object to improve realistic sense during medical training. Specifically, haptic force was produced by calculating related attributes upon surgical instrument touching the target object ${ }^{(6)}$, which facilitates the realistic experiment without risking the life of a real patient. Unfortunately, for ultrasonography, these techniques would suffer from deficiencies inherited from its 
acquisition. Most importantly, patient's movement and low Signal to Noise Ratio (SNR) would prohibit real-time and accurate automatic object extraction at a satisfactory precision. Current applications have thus far been limited to relatively low resolution objects such as segmentation of a left ventricle in echocardiography, etc. This suggests that, until ultrasonography can generate higher quality images, expert intervention is therefore inevitable.

Motivated by this scenario, this paper presents a method which assists interactive surface reconstruction of 3D model from ultrasonographic images. This framework was based on two well established algorithms, i.e., Active Contour and Spherical Harmonics for initial segmentation and 3D surface reconstruction, respectively. Subsequent interactive model editing was permitted by properly organizing relevant data structure so as to optimize local surface modification. The resultant parametric surface model can readily serve as a source for subsequent quantification should require. With interactive editing, any mistake or inaccurate segmentation resulted from compromised image quality, can be amended by medical expert. In the experiment, the proposed scheme was validated against both the synthetic models (a sphere and a pumpkin) and a real fetal image, whereby accuracy analysis and time complexity was reported.

\section{Background}

\subsection{Image segmentation}

Segmentation is a method by which the object of interest is extracted from the image. The method is a primary step toward various medical image analysis and therapeutic intervention schemes, such as left ventricular analysis and brain and cerebral mapping, using MRI, vertebral extraction from CT and fetal diagnosis and therapy by means of an ultrasound sequence. Generally, a single image acquisition contains not only the anatomical structure of interest but also its peripherals, i.e., connective tissue, bone and organs. Furthermore, there are also noise and artifacts present at various degrees, depending on acquiring modality. Medical image segmentation can be divided in to two categories, pixel and parameter based, depending on the objective of the study. This paper focuses primarily on the later, whose main advantage is its ability to offer analytical parameters that can readily be used to derive quantitative attributes, e.g., length, areas, and volumes.
Active Contour: Snake ${ }^{(6)}$ is a simple yet reliable scheme, widely adopted in the literatures. ${ }^{(5)}$ The method extracts boundary of an object based on the minimization of energy, resulted from image forces (external) and curve constraints (internal). ${ }^{(7)}$ It also allows user intervention via adjustable weighting parameters to suite problem at hand. In addition, moving objects can be tracked from an image sequence by incorporating temporal constraints. Detailed treatments on its variants can be found in ${ }^{(8,9)}$ and references therein.

\subsection{Surface Reconstruction}

3D surface model of an object can be reconstructed from segmented entities. Marching cubes ${ }^{(10)}$, for instance, build a network of triangle mesh whose each facet is extracted from 8 -connected pixels using tri-linear interpolation. Since the model contains a set of vertex points, that may be too scattering and noisy to be analyzed. The spherical harmonic function can thus be applied to regularize the mesh entity while retaining its geometry ${ }^{(11,12)}$. Model reconstruction begins with converting each point on a genus-zero surface into corresponding spherical coordinates. Fourier transform is then applied to expand those points with harmonic series on a unit circle. The resultant surface can be obtained from uniformly sampled polar and azimuth angles on the sphere. Different model resolutions can thus be obtained by varying sampling intervals. Several investigations have been carried out based on the idea. Filtering the model based on generic signal processing was proposed in ${ }^{(13)}$, where a 2-manifold mesh was considered as a vector function, i.e., signal, which was then decomposed into a spherical domain by harmonic transforms. Most traditional filtering technique consisting of kernel convolution can then be applied either spatially or in the frequency domain. More specifically, low-, band- or high-pass filters are used to suppress high frequency noise, irregularities or enhance some desired information. Upon building an optimal filter, ${ }^{(14)}$ proposed a sampling scheme, which offers accurate computation on a harmonic and its inverse of a band limit signal. In their work, surface with $\mathrm{L}^{2}$ samples has a band limitation of $\mathrm{L}$ in the harmonic space. Based on the Driscoll and Healy theory, the sampling along longitude axis was taken over by corresponding iso-latitude rings, resulting in variable separation in Spherical Harmonic calculation. Computational complexity and accuracy were validated on the real dataset. Their solution was suitable to models whose band was limited to $10-10^{2}$ (e.g., acoustic, quantum chemistry, and medical imaging) but not much so for higher resolution ones, e.g., those in astrophysics and 
cosmology $\left(10^{2}-10^{3}\right)$. Focusing more into applications, ${ }^{(15)}$ proposed cortical surface reconstruction from MEG data. In this work MEG/ EEG signal was approximated and projected onto a brain surface by spherical harmonic expansion. The neural efficiency could thus be assessed from magnetic and electric fields without having to measure the brain activities on the entire cortical surface. Learning spherical harmonic features (SHF) for 3D face recognition was proposed in ${ }^{(16)}$. In this work, the face model was separated into multi-band frequencies which decomposed facial features into gross shape and fine surface details. The frequency components could then be used in model learning and recognition that are robust against facial expression and occlusion.

\subsection{Interactive Surface Editing}

Thus far, interactive experience could only be achieved by fast calculation of the harmonic expansion. $\mathrm{In}^{(17)}$, the recursive computation of spherical harmonic coefficients of large degree was proposed. In their work, the asymptotic behavior for the rotation coefficients $H_{l}^{m^{\prime} m}(\beta)$ of a large degree $n$ by recursion was studied. It was discovered that despite its instability increasing with $n$, the growth rate of perturbation was sufficiently small that expansion a surface with as large $n$ as $10^{4}$ was feasible. Alternatively, FFT-based algorithm with the complexity of $O\left(n^{2} \log n\right)$ was suggested for $n \leq 10^{3}$ with comparable complexity but slightly lower accuracy.

Laplacian Surface Editing ${ }^{(18)}$ was proposed to manually adjust model to closely resemble the underlying geometry. In this work, geometric details were encoded as an intrinsic property for a vertex. Since Laplacian operator is invariant against rigid transformation and scaling, free-form editing of a model was carried out interactively by merging these geometric data between two model transitions. The sparse linear system can be adopted to speed up the computation. Similarly, the same principle is also applicable for modeling other attributes such as texture, etc. Pointshop $3 \mathrm{D}^{(19)}$ was an interactive system for point-based surface editing. The proposed scheme was able to interactively edit the surface of interest by cleaning, texturing, sculpting, carving, filtering and resampling. It consisted of 2 processes, which are point cloud parameterization with low distortion and continuous resampling method. In this method, $2 \mathrm{D}$ image operations were used as editing tools without relying on intermediate tessellation. It was suggest that the system is suitable for a low cost 3D scanners and rendering pipelines.

Particularly in medical image analysis, segmentation is often entailed with supervised adjustment. While doing so on the slice-by-slice basis is time consuming, editing surface in $3 \mathrm{D}$ is also prone to inter-observer influence. Thus, editing surface in other spaces was suggested. In ${ }^{(20)}$, real-time 3D interactive segmentation of ultrasound data by the B-Spline Explicit Active Surface (BEAS) for recovering an object from volumetric data was proposed. BEAS reduced the overhead from interactive process while maintaining local accuracy and robustness. Manipulating surface by means of B-Spline was also proposed in ${ }^{(21)}$. In their work, model curvature was directly adjusted along the iso-parametric curves at a specified location, provided unit tangent and unit normal at that point, without having to deal with respective control points (knots). Resultant surface was then converted from this edited curvature by iterative repositioning. However, its main drawbacks include the inability work with a surface of arbitrary representation and that bumps may occur.

Although the spectral method has been widely adopted for surface reconstruction, its computational complexity is relatively high of $\mathrm{O}\left(\mathrm{N}^{3}\right)$, due to the evaluation of Legendre coefficients for arbitrary cut-off frequency $\mathrm{N}$. $\mathrm{In}^{(22)}$, a fast spherical harmonic transform was thus presented, whereby Legendre coefficients were approximated by polynomial transform that could be sped up by Fast Multiple Method (FMM). With this technique the complexity was reduced to $\mathrm{O}\left(\mathrm{N}^{2} \log \mathrm{N}\right)$. There are however shortcomings need to be addressed, e.g., the principal of FMM remains unclear, the approximation requires Chebyshev polynomial and singular value decomposition to improve its efficiency, non-uniform point are left untreated, it has large storage requirement, the effects of different choice of split, sampling and division points on approximation error were not assessed. Similarly, in ${ }^{(23)}$ a fast directional spatial localized spherical harmonic transform (SLSHT) in a spatio-spectral domain was present. Prior to spherical harmonic transformation, an asymmetric windows function was used localize directional feature in spatio-temporal domain. The corresponding inversion was then defined by an alternative harmonic formulation where factoring of rotation approach and FFT were adopted. With a band limit signal $\mathrm{L}_{\mathrm{f}}$, the SLSHT complexity and that of its 
inverse are given by $O\left(L_{f}^{3} L_{h}^{2}+L_{f}^{2} L_{h}^{4}\right)$ and $O\left(L_{f}^{4} L_{h}^{3}\right)$.

\section{Material and methods}

In this work, a set of contours were segmented from the ultrasound images resided on arbitrary 2D planes by using an Active Contour. The resultant cloud points extracted from these contours formed the surface outline which was then fitted with the spherical harmonic function. The 3D reconstructed model could be edited interactively with our proposed localizing scheme. Fig. 1. depicts the diagram summarizing our framework.
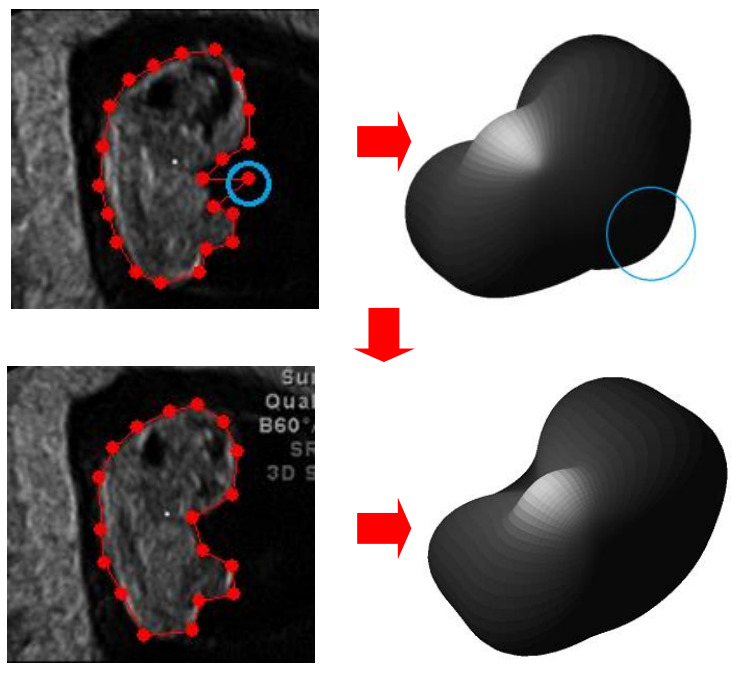

Fig. 1. The flow diagram of the proposed framework

\subsection{Image Segmentation: Active Contour}

Segmenting the object of interest used the basic Active Contour algorithm or Snake implement with a fast greedy search strategy. The formulation of the Snake energy to be optimized for each object contour is given by:

$$
E=\int\left(\alpha E_{\text {continuity }}+\beta E_{\text {curvature }}+\gamma E_{\text {image }}\right) d s
$$

where $\mathrm{E}_{\text {continuity }}$ and $\mathrm{E}_{\text {curvature }}$ monitor control points interval and their curvature, respectively. They are expressed by

$$
\begin{aligned}
& E_{\text {continuity }}=\left(\bar{d}-\left|v_{i}-v_{i-1}\right|\right) \\
& E_{\text {curvature }}=\left|v_{i-1}-2 v_{i}+v_{i+1}\right|^{2}
\end{aligned}
$$

The $\mathrm{E}_{\text {image }}$ term was derived from an image gradient of the underlying pixel. It was simply calculated by the magnitude of Sobel kernel response. Upon greedy search, 8connected region search for a point with the minimum overall energy according to (1) to which the respective control point was moved in the next iteration. The process was repeated for each control point in turn until convergence. Fig.2. shows the example of segmented fetal contours from 3 selected planes.

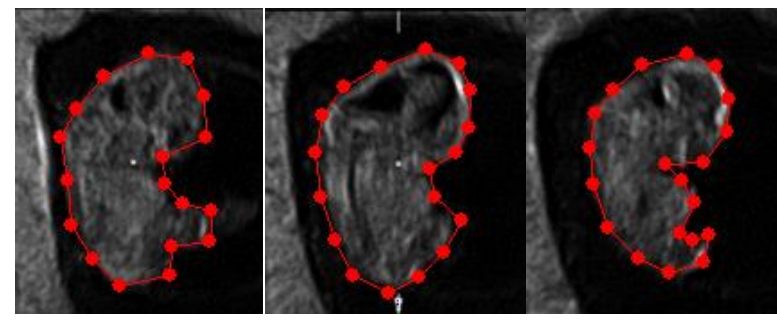

Fig.2. Examples of segmented fetal contour

\subsection{Surface Reconstruction: Spherical Expansion}

Since each contour was extracted from an arbitrary 2D plane residing on a 3D space, its control points had also 3D coordinates and thus all the segmented contours formed a set of cloud points outlining a surface model. These points was expanded for each coordinate by Spherical Harmonic expansion, with $\theta \in[0,2 \pi]$ and $\phi \in[0, \pi]$,

$$
x(\theta, \phi)=\sum_{l=0}^{\infty} \sum_{m=-l}^{l} c_{l}^{m} Y_{l}^{m}(\theta, \phi)
$$

where the coefficient $c_{l}^{m}$ for each order $\mathrm{m}, \mathrm{l}$ was calculated by the expression: $c_{l}^{m}=\left(Z^{T} Z\right)\left(Z^{T} V\right)$, where $\mathrm{V}$ is a vector containing original vertices in a spherical coordinate system and $Z_{l, m}=Y_{l}^{m}\left(\theta_{i}, \phi_{i}\right)$. The spherical harmonic function on a sphere is defined as follow: 


$$
\begin{aligned}
Y_{l}^{m}(\theta, \phi) & =\sqrt{\frac{2 l+1}{4 \pi} \frac{(l-|m|) !}{(l+|m|) !}} P_{l}^{|m|}(\cos \theta) e^{i|m| \phi} \\
P_{l}^{m}(x) & =\frac{(-1)^{m}}{2^{l} l !}\left(1-x^{2}\right)^{\frac{m}{2}} \frac{d^{m+1}}{d x^{m+1}}\left(x^{2}-1\right)^{l}
\end{aligned}
$$

Expressions for $\mathrm{y}$ and $\mathrm{z}$ could be similarly computed.

\subsection{Image Segmentation: Active Contour}

Since the image quality of ultrasonogrphy is relatively low compared to other modalities, the segmented object may not perfectly coincide with underlying boundaries. Moreover, the segmentation was done independently for each of the involving planes, which during the scan, might be misaligned due to subject and probe motion, resulting in inconsistent 3D model. Therefore, manual editing is often inevitable. However, as discussed above, reconstructing the entire harmonic surface for each local point editing would be impedingly expensive. To address this issue, this paper hence suggests data structure manipulation for an efficient evaluation of $C_{l}^{m}$ coefficients during local editing. The flow of interactive model reconstruction and editing is described in Fig.3.

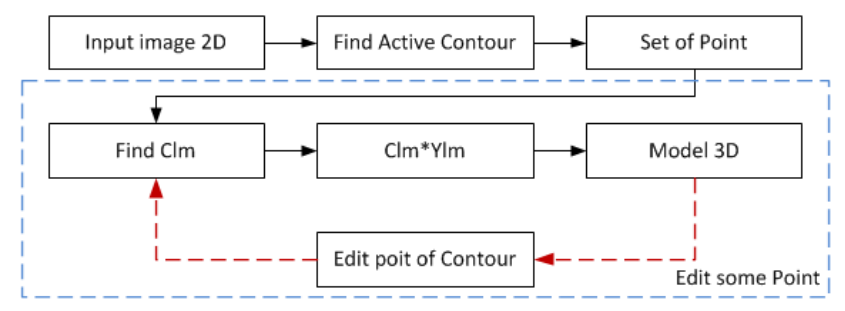

Fig.3. Interactive model reconstruction and editing

Once the initial surface was reconstructed from the cloud points, instead of re-evaluating all the expressions in (3) and (4), some redundancy could be exploited during local point adjustment. Given a set of points $\mathbf{p}_{\mathrm{i}}(x(\theta, \phi), y(\theta, \phi), z$ $(\theta, \phi))$ where for $i=0 \ldots n-1$, their respective harmonic functions at each order $l$ and $m$, were stored in the format as depicted in Fig.4.

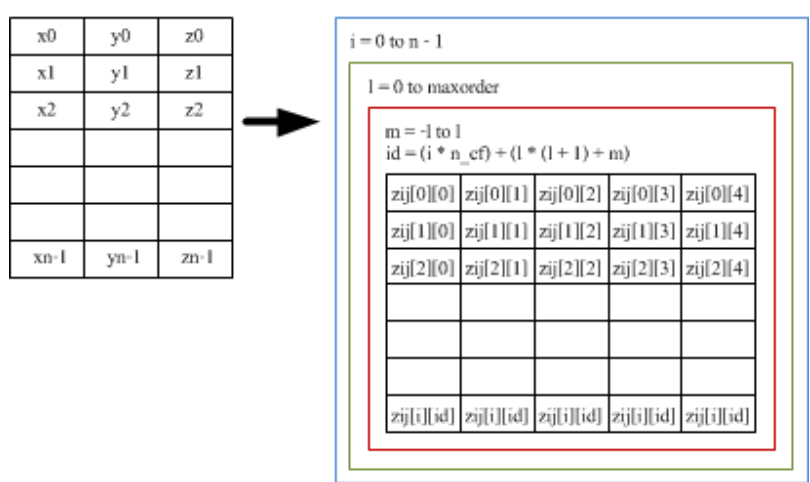

Fig.4. Data structure that stores harmonic function $Z$.

Following the definition in equation (3), $C_{l}^{m}$ coefficients were computed by multiplication of 2 terms involving $Z$ and $V$, i.e. $c_{l}^{m}=\left(Z^{T} Z\right)\left(Z^{T} V\right)$, whose diagram is illustrated in

Fig.5. Since the most computationally expensive step is the calculation of the harmonic function $\mathrm{Z}$, its indexing scheme in relation to a given point $\mathbf{p}_{\mathrm{k}}(\mathrm{x}, \mathrm{y}, \mathrm{z})$ to be edited was investigated. It was revealed that, a systematic indexing scheme was feasible for a few members of the $\mathrm{Z}$, whose the new evaluation was required. For a given $k^{\text {th }}$ point, these members are illustrated in Fig.5.

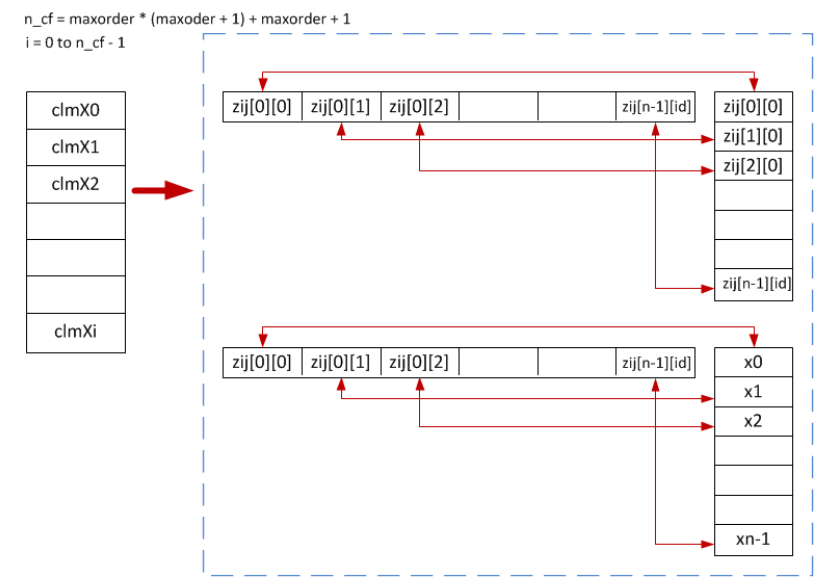

Fig. 5. Calculation of the coefficients $C_{l}^{m}$ for $x(\theta, \phi)$

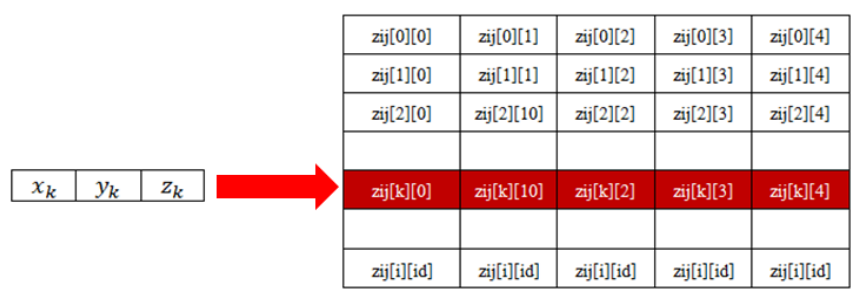


Fig. 6. Systematic indexing scheme for Z, given $k$. The cells highlighted indicate those need modification.

Accordingly, if a $k^{\text {th }}$ point was moved be the effected $\mathrm{Z}$ values associated with its previous location were removed from the $C_{1}{ }^{m}$ and replaced with those associated with the new one, as given in (5).

$$
\begin{aligned}
c \operatorname{lm} x_{\text {new }} & =c \operatorname{lm} x_{\text {old }} \\
& -\left(o_{-} z i j_{k} * o_{-} z i j_{k}\right)\left(o_{-} z i j_{k} * o_{-} x_{k}\right) \\
& +\left(n_{-} z i j_{k} * n_{-} z i j_{k}\right)\left(n_{-} z i j_{k} * n_{-} x_{k}\right)
\end{aligned}
$$

The evaluation for $y$ and $\mathrm{z}$ coordinates could be similarly calculated. The graphical representation of the updating scheme is showed in Fig. 7.

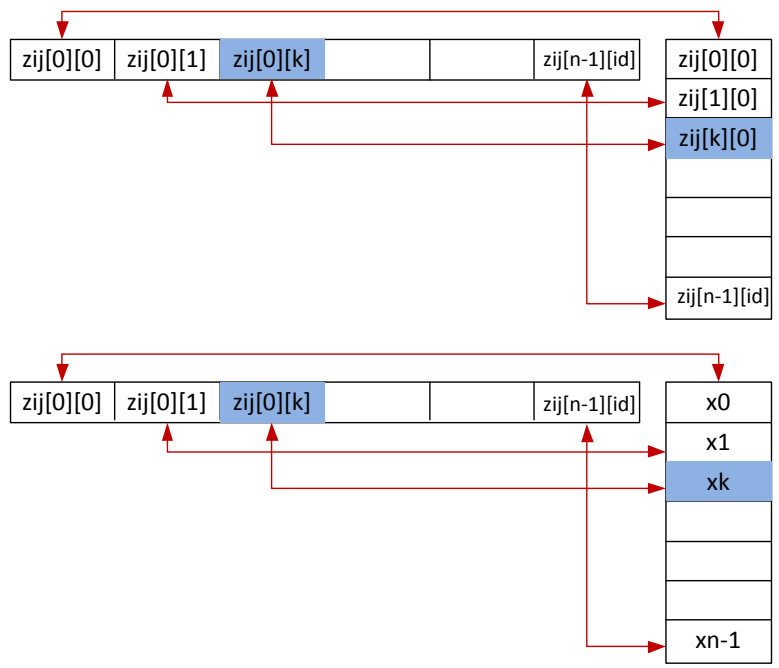

Fig. 7. Updating scheme, given $k$.

Because only a fraction of the entire $Z$ structure, whose expression involved rather expensive Legendre polynomial calculation, needs to be recomputed per each modification, a tremendous time was conserved, resulting in interactive experience in the user perspective.

\section{Experiments and Result}

In order to assess the proposed technique, 2 synthetic data, i.e. a sphere (420 points) and a pumpkin (660 points), as well as 1 fetal shape (175 points) that was segmented from ultrasound images were employed in the experiment. The obtained results are described as follow.

\subsection{Accuracy}

For each data set, the surface was reconstructed with varying order (2, 3 and 5), where the original cloud points were compared with those approximated with respective Spherical Harmonic. Their errors in pixels and percents were

\begin{tabular}{|c|c|c|c|c|c|}
\hline Shape & $\begin{array}{l}\text { Data } \\
\text { (Point) }\end{array}$ & $\begin{array}{l}\text { Avg.Dist } \\
\text { (Pixel) }\end{array}$ & Order & $\begin{array}{l}\text { Error } \\
\text { (Pixel) }\end{array}$ & $\begin{array}{l}\text { Error } \\
(\%)\end{array}$ \\
\hline \multirow{3}{*}{ Circle } & \multirow{3}{*}{420} & \multirow{3}{*}{49.35289} & 2 & 0.82801 & 1.67773 \\
\hline & & & 3 & 0.81509 & 1.65156 \\
\hline & & & 5 & 0.57012 & 1.15519 \\
\hline \multirow{3}{*}{ Pumpkin } & \multirow{3}{*}{660} & \multirow{3}{*}{65.59584} & 2 & 10.04605 & 15.31508 \\
\hline & & & 3 & 1.53241 & 2.33614 \\
\hline & & & 5 & 0.71881 & 1.09582 \\
\hline \multirow{3}{*}{ Fetal } & \multirow{3}{*}{175} & \multirow{3}{*}{54.101545} & 2 & 9.54829 & 17.64884 \\
\hline & & & 3 & 6.19768 & 11.45565 \\
\hline & & & 5 & 4.92446 & 9.10225 \\
\hline
\end{tabular}
reported in Table 1.

Table 1 Surface reconstruction accuracy.

Particularly for the 3D fetal shape, surface reconstruction accuracy was dependent not only on the harmonic function but also the initial Active Contour segmentation. The results in Table 2. show reconstruction errors given initial shape segmentations from one observers with different estimates (Error 1 to 4), and their average.

Table 2. Intra-observer surface reconstruction variability of fatal shape.

\begin{tabular}{|c|c|c|c|c|c|c|}
\hline \multirow{2}{*}{ Shape } & Order & $\begin{array}{c}\text { Error1 } \\
(\%)\end{array}$ & $\begin{array}{c}\text { Error2 } \\
(\%)\end{array}$ & $\begin{array}{c}\text { Error3 } \\
(\%)\end{array}$ & $\begin{array}{c}\text { Error4 } \\
(\%)\end{array}$ & $\begin{array}{c}\text { ErrorAvg. } \\
(\%)\end{array}$ \\
\hline \multirow{3}{*}{ Fetal } & 2 & 16.52334 & 17.82908 & 17.44493 & 17.64884 & 17.36155 \\
\cline { 2 - 7 } & 3 & 10.43020 & 11.06574 & 11.08798 & 11.45565 & 11.00989 \\
\cline { 2 - 7 } & 5 & 7.853006 & 8.190991 & 8.147735 & 9.102259 & 8.32349 \\
\hline
\end{tabular}


Table 3. shows reconstruction errors given initial shape segmentations from 4 different observers (Error 1 to 4 ) each with one estimate, and their average.

Table 3. Inter-observer surface reconstruction variability of fatal shape.

\begin{tabular}{|c|c|c|c|c|c|c|}
\hline Shape & Order & $\begin{array}{c}\text { Error1 } \\
(\%)\end{array}$ & $\begin{array}{c}\text { Error2 } \\
(\%)\end{array}$ & $\begin{array}{c}\text { Error3 } \\
(\%)\end{array}$ & $\begin{array}{c}\text { Error4 } \\
(\%)\end{array}$ & $\begin{array}{c}\text { ErrorAvg. } \\
(\%)\end{array}$ \\
\hline \multirow{3}{*}{ Fetal } & 2 & 16.71039 & 17.11463 & 17.68332 & 17.69965 & 17.30200 \\
\cline { 2 - 7 } & 3 & 10.74449 & 10.25064 & 11.77549 & 11.35397 & 11.03115 \\
\cline { 2 - 7 } & 5 & 7.69873 & 7.73562 & 9.06938 & 8.36247 & 8.21655 \\
\hline
\end{tabular}

\subsection{Time Efficiency}

Table 4. list the time (in sec.) that was required for the entire surface reconstruction compared with that when using our selective updating scheme. It is evident that the time was significantly reduced and it is anticipated that the reduction ratio would be increase as the number of points increase.

Table 4.Times required for total surface reconstruction and selective updating for each models.

\begin{tabular}{|c|c|c|c|c|c|c|}
\hline \multirow[b]{2}{*}{ Shape } & \multirow{2}{*}{$\begin{array}{l}\text { Data } \\
\text { (Point) }\end{array}$} & \multirow[b]{2}{*}{ Order } & \multicolumn{4}{|c|}{ Times (sec.) } \\
\hline & & & $\begin{array}{c}\text { All } \\
\text { process }\end{array}$ & 1 point & 2 point & 3 point \\
\hline \multirow{3}{*}{ Circle } & \multirow{3}{*}{480} & 2 & 0.065 & 0.002 & 0.003 & 0.005 \\
\hline & & 3 & 0.076 & 0.003 & 0.005 & 0.007 \\
\hline & & 5 & 0.130 & 0.012 & 0.020 & 0.030 \\
\hline \multirow{3}{*}{ Pumpkin } & \multirow{3}{*}{660} & 2 & 0.076 & 0.002 & 0.003 & 0.005 \\
\hline & & 3 & 0.079 & 0.003 & 0.005 & 0.008 \\
\hline & & 5 & 0.139 & 0.012 & 0.020 & 0.030 \\
\hline \multirow{3}{*}{ Fetal } & \multirow{3}{*}{191} & 2 & 0.061 & 0.002 & 0.003 & 0.005 \\
\hline & & 3 & 0.074 & 0.003 & 0.005 & 0.007 \\
\hline & & 5 & 0.125 & 0.010 & 0.017 & 0.030 \\
\hline
\end{tabular}

It is clearly seen that the reconstruction errors decrease as the harmonic increase, at obviously the price of increase computational time. The reported values conformed well to the graphical illustration shown in Fig. 8. This calls for the selective indexing scheme proposed in this paper to reduce the computational burden.

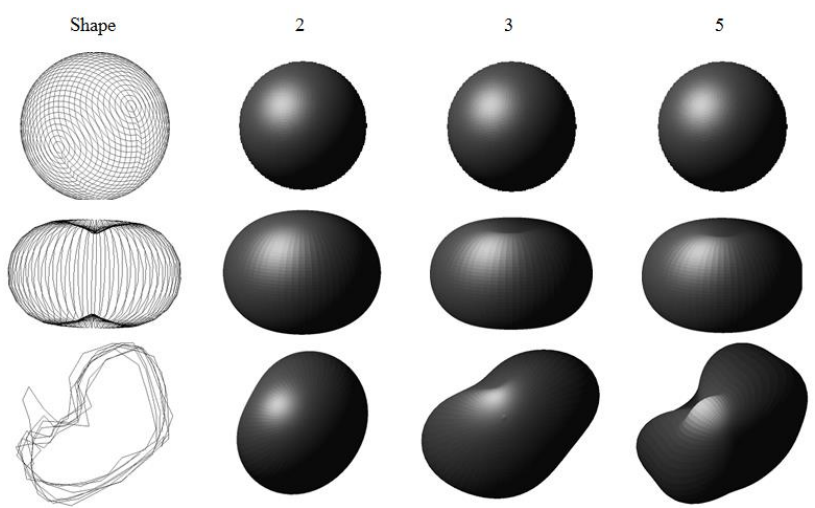

Fig. 8. Graphical illustration of the reconstructed surfaces at varying harmonic orders.

\section{Conclusion}

Reconstruction of a 3D surface model from a set of 2D images involved segmenting a planar object from a set of $2 \mathrm{D}$ images. If the object planes are parallel any systematic triangulation techniques such as Marching Cubes may be employed. On the contrary, if those planes sample thro ugh the 3D space at differing angles as in the case of ultrasound modality, a more time consuming surface reconstruction, such as spherical harmonic function was needed. In order to accurately reconstruct the surface accurately, a high numbers of harmonic order and hence large computation time were required.

Conventionally, in case where the underlying points need to be modified, the entire reconstruction process must be completed. This paper thus proposed a systematic indexing scheme which selectively updated harmonic function as per necessary. Experiment numerical results show a promising reduction in time-complexity burden. An interactive surface segmentation and editing framework developed based on this technique could offer responsive feeling to the user.

\section{References}

(1) R.A. Robb :"VR Assisted Surgery Planning”, IEEE Eng. in Med. Bio. Mag., Vol. 15, No. 2, pp. 60-69, DOI: 10.1109/51.486720,Mar/Apr 1996 
(2) Haubner, M. et. al. :"Virtual reality in medicine-computer graphics and interaction techniques", IEEE Trans. Info. Tech. Biomed., Vol.1, No. 1, pp. 61-72, DOI: 10.1109/TITB.2006.884359, Mar. 1997

(3) Tong-Yee Lee, et. al. :"Interactive 3-D Virtual Colonoscopy System”, IEEE Trans., Info. Tech. Biomed., Vol. 3, No.2, pp.139-150, DOI: 10.1109/4233.767089, June 1999

(4) Panchaphongsaphak, B. et. al. :"Three-Dimensional Touch Interface for Medical Education", IEEE Trans. Info. Tech. Biomed., Vol.11, No. 3, pp. 251-263, DOI: 10.1109/TITB.2006.884359, May 2007

(5) Marián Bakoš, "Active Contours and their Utilization at Image Segmentation", 2007

(6) Micheal Kass, Andrew Witkin and DemetriTozopoulos, "Snakes: Active contour models" International Journal of Computer Vision, Volume 1, Issue 4, pp. 321-331, January 1988

(7) Sandra V. B. Jardim, Mário A. T. Figueiredo, "Automatic Contour Estimation In Fetal Ultrasound Images”, 2003

(8) Hill, C. R., Bamber, J. C., and ter Haar, G. R., Jeongju Choi, Jong Shik Kim :"Physical Principles of Medical Ultrasonics", 2nd John Wiley\&Sons, 2002.

(9) K.M.Lam and H Yan, "Fast greedy algorithm for active contours", 1993

(10) William E. Lorensen and Harvey E. Cline, “ Marching cubes: A high resolution 3D surface construction algorithm”, 1987

(11) W.H. Press, B.P. Flannery, S. A. Teukolsky, and W. Vetterling, 1988 : "Spherical Harmonics." §6.8 in Numerical Recipes in FORTRAN", The Art of Scientific Computing, 2nd ed. Cambridge, England: Cambridge University Press, pp. 246-248, 1992

(12) Sittichai P., Paramate H., Saowapak S.:"Multi-resolution ICP using real-valued spherical harmonic expansion and its application in computer-aided dental", The 3rd International Symposium on Biomedical Engineering (ISBME 2008), 2008

(13) Kun Zhou, Hujun Bao, Jiaoying Shi, “3D surface filtering using spherical harmonics", Computer-Aided Design 36, pp. 363-375, 2004

(14) Zubair Khalid, Rodney A. Kennedy, and Jason D. McEwen : "An Optimal-Dimensionality Sampling Scheme on the Sphere with Fast Spherical Harmonic Transforms", arXiv1403:4661v2 [cs.IT], 23 July 2014.

(15) Jose D. L' opez, Luzia Troebinger, Will Penny, Jairo J. Espinosa, and Gareth R. Barnes ': "Cortical surface reconstruction based on MEG data and spherical harmonics", 35th Annual International Conference of the IEEE EMBS, 3 7 July, 2013.
(16) Peijiang Liu, Yunhong Wang, Di Huang, Zhaoxiang Zhang and Liming Chen : "Learning the Spherical Harmonic Features for 3-D Face Recognition", IEEE TRANSACTIONS ON IMAGE PROCESSING, VOL. 22, NO. 3, pp. 914-925, MARCH 2013

(17) Nail A. Gumerov and Ramani Duraiswami : "Recursive computation of spherical harmonic rotation coefficients of large degree", arXiv1403:7698v1 [cs.NA], 30 March 2014.

(18) O. D. Cohen-Or, Y. Lipman1, M. Alexa, C. Rössl and H.-P. Seidel : "Laplacian Surface Editing", Eurographics Symposium on Geometry Processing, 2004

(19) Matthias Zwicker, Mark Pauly, Oliver Knoll, Markus Gross and ETH Zürich : "Pointshop 3D: An Interactive System for Point-Based Surface Editing”, pp.322-329.

(20) Daniel Barbosa, Brecht Heydea, Maja Cikes, Thomas Dietenbeck, Piet Claus, Denis Friboulet, Olivier Bernard, Jan D'hoogea : "Real-time 3D interactive segmentation of echocardiographic data through user-based deformation of Bspline explicit active surfaces", Computerized Medical Imaging and Graphics 38, pp.57-67, 2014

(21) Yuki Kineri, Shuhei Endo, Takashi Maekawa : "Surface design based on direct curvature editing", Computer-Aided Design 55, pp.1-12, 2014

(22) Reiji suda and Masayasu Takami : "A fast spherical harmonics transform algorithm", mathematics of computation, Vol. 71, No. 238, Pages 703-715, Nov 28, 2001

(23) Zubair Khalid, Rodney A. Kennedy, Salman Durrani, Parastoo Sadeghi, Yves Wiaux and Jason D. McEwen : "Fast Directional Spatially Localized Spherical Harmonic Transform", IEEE TRANSACTIONS ON SIGNAL PROCESSING, VOL. 61, NO. 9, pp.2192-2203 , MAY 1, 2013 